\title{
The TRAIL to cancer therapy: hindrances and potential solutions
}

\begin{abstract}
Apoptosis is an ordered and orchestrated cellular process that occurs in physiological and pathological conditions. Resistance to apoptosis is a hallmark of virtually all malignancies. Despite being a cause of pathological conditions, apoptosis could be a promising target in cancer treatment. Tumor necrosis factor (TNF)-related apoptosis-inducing ligand (TRAIL), also known as Apo-2 ligand (Apo2L), is a member of TNF cytokine superfamily. It is a potent anti-cancer agent owing to its specific targeting towards cancerous cells, while sparing normal cells, to induce apoptosis. However, resistance occurs either intrinsically or after multiple treatments which may explain why cancer therapy fails. This review summarizes the apoptotic mechanisms via extrinsic and intrinsic apoptotic pathways, as well as the apoptotic resistance mechanisms. It also reviews the current clinically tested recombinant human TRAIL (rhTRAIL) and TRAIL receptor agonists (TRAs) against TRAIL-Receptors, TRAIL-R1 and TRAIL-R2, in which the outcomes of the clinical trials have not been satisfactory. Finally, this review discusses the current strategies in overcoming resistance to TRAIL-induced apoptosis in pre-clinical and clinical settings.
\end{abstract}

Keyword: TRAIL; Cancer therapy; Resistance 